\title{
Biomechanical Analysis of Unstable Osteochondral Fragment Fixation Using Three Different Techniques: Osteochondral Plug, Bioabsorbable Pin, and Suture Anchor with Tape
}

\author{
Shizuka Sasaki, M.D., Shohei Yamauchi, M.D., Eiji Sasaki, M.D., Yuka Kimura, M.D., \\ Akane Takahashi, M.D., and Yasuyuki Ishibashi, M.D.
}

Purpose: To compare the initial fixation strength of osteochondral fragment fixations using osteochondral plugs, bioabsorbable pins, and knotless suture anchors. Methods: Eighteen fresh-frozen immature (6 month old) porcine knees were used. An osteochondral fragment, cut from the articular surface of the medial femoral condyle to achieve a thickness of $5 \mathrm{~mm}$, was used to mimic the unstable osteochondral fragment. It was fixed using three techniques, including two osteochondral plugs (osteochondral plug group), four full-threaded poly L-lactic acid pins (bioabsorbable pin group), and three suture anchors with a 2-0 tape (suture anchor group). Tensile loads at displacements of 1 and $2 \mathrm{~mm}$ and ultimate failure load were measured at a cross-head speed of $100 \mathrm{~mm} / \mathrm{min}$, and the variables of the three groups were compared statistically using a one-way ANOVA with Tukey's honestly significant difference test. Results: There was no significant difference in the tensile load to achieve $1-\mathrm{mm}$ displacement. The load to achieve 2 -mm displacement and the ultimate failure load were significantly greater in the suture anchor group than the osteochondral plug group and the bioabsorbable pin group. Conclusions: Single-pull destructive testing of a fixed articular osteochondral fragment with the force perpendicular to the articular surface, demonstrated no statistical difference in the tensile load to achieve l-mm displacement, but the load to achieve $2-\mathrm{mm}$ displacement was significantly greater for the three suture anchorinterlocking 2-0 tape constructs than the dual osteochondral plug fixation and the four bioabsorbable pin fixation constructs. Additionally, the three suture anchor-interlocking 2-0 tape construct's mean single-pull failure load was greater than other two fixation procedures. Clinical Relevance: To achieve osteochondral fragment union, sufficient fixation strength is critical. However, the initial fixation strength of osteochondral plugs, bioabsorbable pins, and knotless suture anchors for unstable osteochondral lesions remains unclear.

\section{Introduction}

$\mathbf{T}$ he treatment strategy for knee osteochondral lesions (OCLs), including osteochondral dissecans (OCD) and osteochondral fracture, depends on the

From the Department of Orthopaedic Surgery, Hirosaki University Graduate School of Medicine, Hirosaki, Japan.

The authors report no conflicts of interest in the authorship and publication of this article. Full ICMJE author disclosure forms are available for this article online, as supplementary material.

Received March 1, 2021; accepted August 25, 2021.

Address correspondence to Shizuka Sasaki, M.D., Department of Orthopaedic Surgery, Hirosaki University Graduate School of Medicine, Zaifu-cho 5, Hirosaki, Aomori 036-8562, Japan.E-mail:ss422412@rmail.plala.or.jp

(C) 2021 THE AUTHORS. Published by Elsevier Inc. on behalf of the Arthroscopy Association of North America. This is an open access article under the CC BY-NC-ND license (http://creativecommons.org/licenses/by-nc-nd/4.0/).

2666-061X/21315

https://doi.org/10.1016/j.asmr.2021.08.019 stability or quality of the osteochondral fragment. OCL treatment aims to restore articular surface congruity and prevent secondary osteoarthritis and persistent pain. If the osteochondral fragment has sufficient subchondral bone quality, osteochondral fragment fixation is a suitable surgical procedure for unstable OCL.

Berlet et al. ${ }^{1}$ reported a fixation technique using a cylindrical autogenous osteochondral plug. Using this technique for unstable knee OCD, Miura et al. ${ }^{2}$ reported good clinical outcomes with a minimum 2-year follow-up. Although this procedure has the advantage of biological fixation, the possibility of donor site morbidity cannot be ignored.

Bioabsorbable pin fixation reportedly leads to good clinical outcomes and histological healing. ${ }^{3-7}$ The benefit of this procedure is that it avoids the risk of donor site morbidity. Because of the same advantage, it 

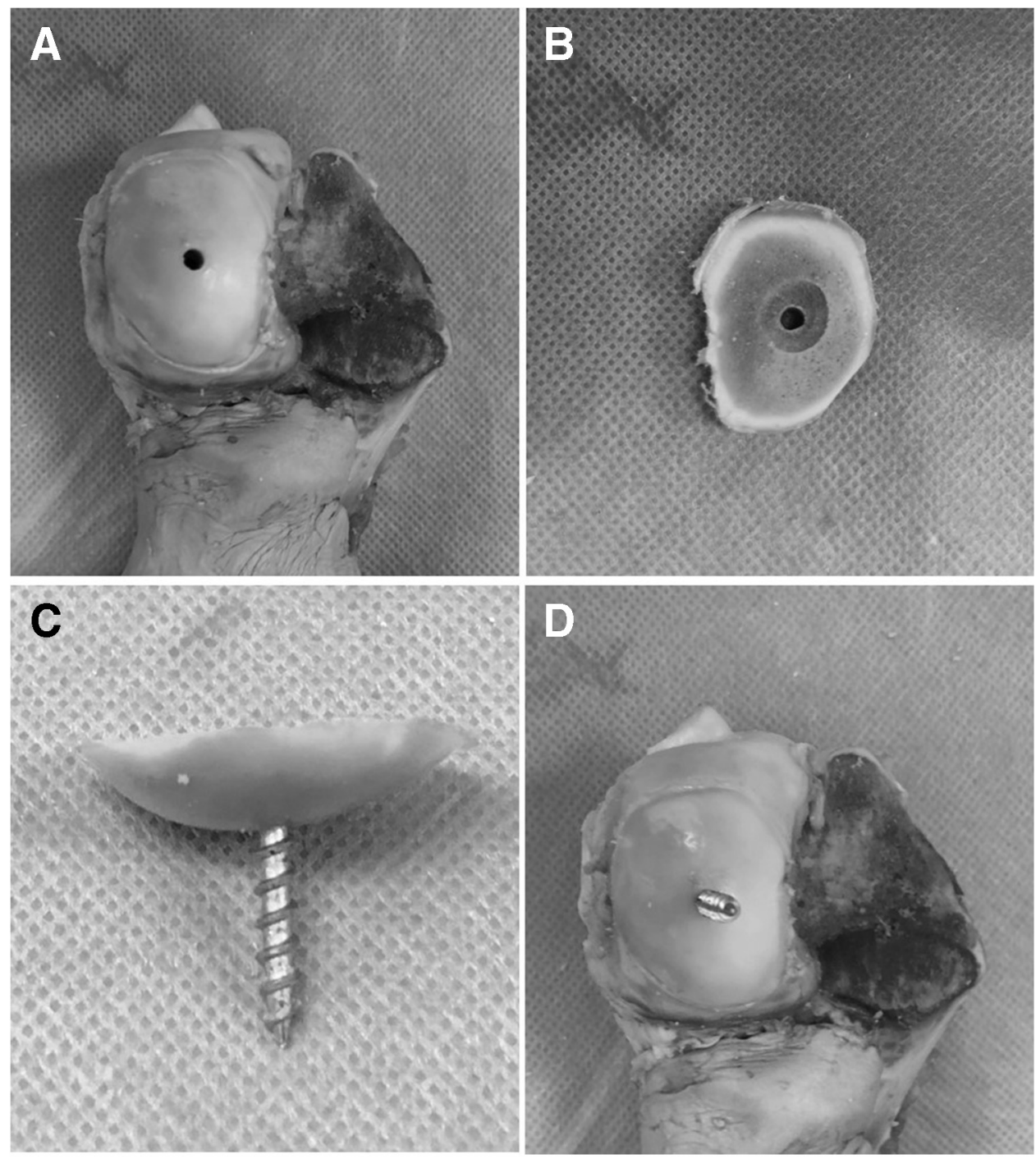

Fig 1. The medial femoral condyle was cut $5 \mathrm{~mm}$ deep ( $\mathrm{A}$ and $\mathrm{B}$ ), and the metal screw that was inserted in the osteochondral fragment was directed perpendicular to the articular surface $(C)$, so that the osteochondral fragment could be reduced anatomically and clamped during tensile load testing, with tensile load applied perpendicular to the osteotomy plane (D). has been reported in recent years that a knotless suture anchor is useful for osteochondral fragment fixation. ${ }^{8-10}$ This technique can provide a wider compression force distribution from the suture bridge configuration. In addition, this procedure has the advantages that the anchor is completely buried under the cartilage, and the implant does not need to be removed.

To achieve osteochondral fragment union, sufficient fixation strength is a seemingly critical factor. However, the initial fixation strength of these surgical procedures remains unclear. The purpose of this study was to compare the initial fixation strength of osteochondral fragment fixations using osteochondral plugs, bioabsorbable pins, and knotless suture anchors. The hypothesis was that the initial fixation strength of the osteochondral fragment fixation with a knotless suture anchor is stronger than that of other previous procedures.

\section{Methods}

Eighteen fresh-frozen immature (6-month-old) porcine knees were used in this study. This study was approved by institutional review board of Hirosaki University (no. 2011-199). Specimens were stored at $-20^{\circ} \mathrm{C}$ and then thawed at room temperature for 24 $\mathrm{h}$ before testing. All soft tissue structures were removed to expose the joint surface of the distal femur. The proximal part of the femur was placed in a mold of polymethylmethacrylate to rigidly grip testing fixtures. An osteochondral fragment was used to mimic an unstable osteochondral fragment, which was cut from the articular surface of the medial femoral condyle to achieve a thickness of $5 \mathrm{~mm}$ (Fig 1). Osteochondral fragments had an anteroposterior diameter of $30.4 \pm 2.7$ $\mathrm{mm}$ and a transverse diameter of $22.5 \pm 1.7 \mathrm{~mm}$. Specimens were divided into three groups of six knees each based on fixation techniques. After anatomical 

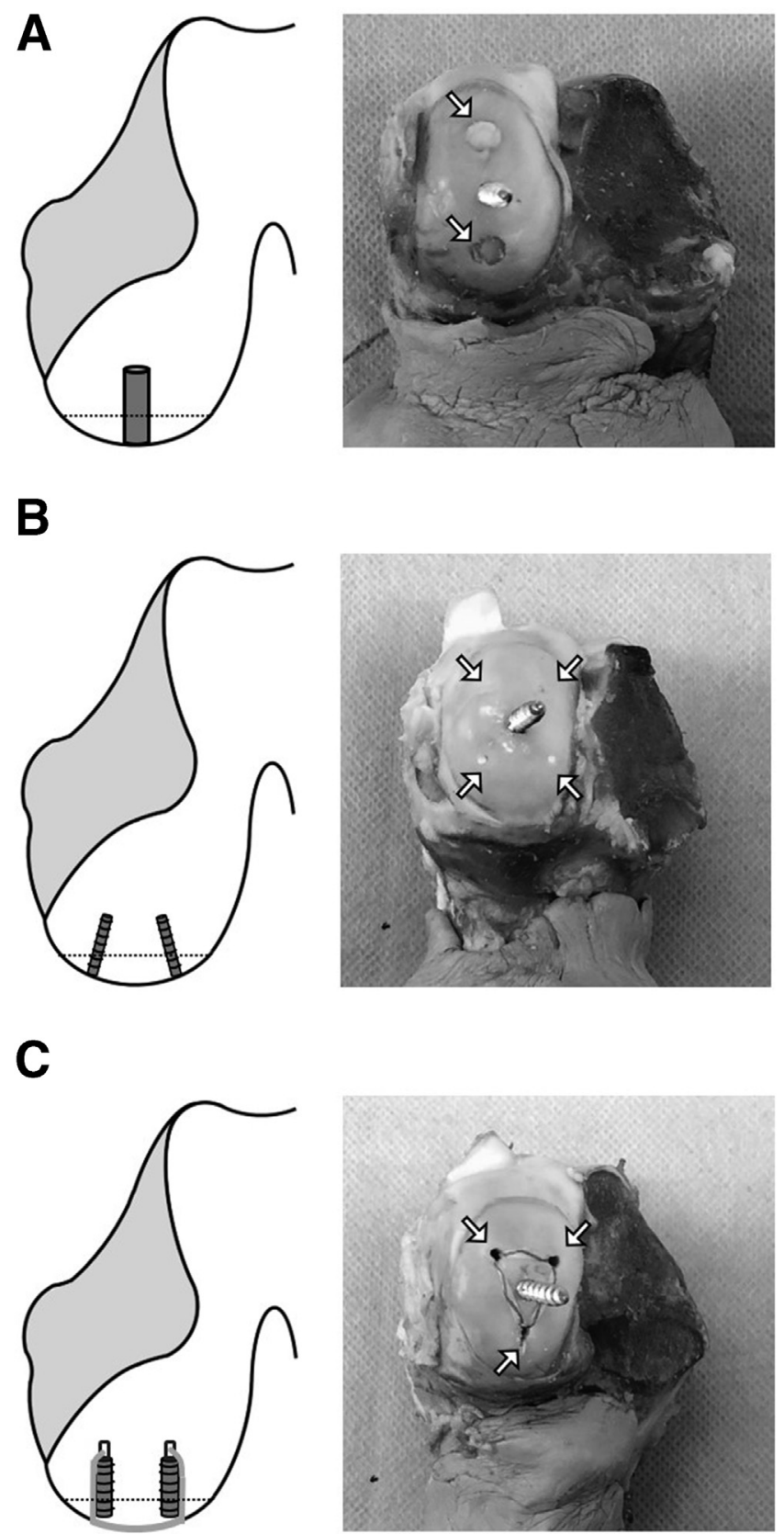

Fig 2. The fixation scheme and photo showing the procedure to fix osteochondral fragment created by cutting the articular surface (dotted line) of the porcine femoral condyle. The osteochondral fragment was fixed with two osteochondral plugs (A), with four full-threaded bioabsorbable pins (B), and with three suture anchors with 2-0 tape $(\mathrm{C})$. The location of the osteochondral plug and device is indicated by arrows.

reduction, the osteochondral fragments were fixed using three different techniques. The number of fixation devices in each group was the same for clinical osteochondral fragments of the same size.

In the osteochondral plug fixation group (osteochondral plug group), ${ }^{1}$ the osteochondral fragment was fixed with two osteochondral plugs $(6.0 \times 15 \mathrm{~mm})$ harvested from the lateral femoral condyle in the same knee. For osteochondral grafting, the Osteochondral Autograft Transfer System (OATS; Arthrex, Naples, FL) was used. Press-fit osteochondral graft implantation was achieved by inserting an osteochondral graft of 6.0 $\mathrm{mm}$ in diameter obtained from the donor site into a smaller recipient hole of $5.0 \mathrm{~mm}$ in diameter. A tube harvester of $6.0 \mathrm{~mm}$ in diameter was set at a depth of 15 $\mathrm{mm}$ in the bone; the cylindrical osteochondral plug was then detached at the base of the tube by rotating the tube harvester $90^{\circ}$. At the recipient site, the recipient hole was created at a depth of $15 \mathrm{~mm}$ using a tube harvester of $5.0 \mathrm{~mm}$ in diameter. The obtained osteochondral plug was inserted into the recipient hole directly from the tube harvester (Fig 2A). During bioabsorbable pin fixation, the osteochondral fragment was fixed with four full-threaded poly L-lactic acid (PLLA) pins $(1.5 \times 20 \mathrm{~mm}$, Takiron Co., Osaka, Japan) after drilling a hole of $2.0 \mathrm{~mm}$ in diameter (bioabsorbable pin group) (Fig 2B). During knotless suture anchor fixation, the osteochondral fragment was fixed with three PushLock anchors $(2.4 \times 11.3 \mathrm{~mm}$, Arthrex, Naples, FL) with a 2-0 Mini SutureTape (Arthrex, Naples, FL) (suture anchor group). The suture anchor consists of a radiolucent body and a separate eyelet made of polyetheretherketone (PEEK) (Fig 2C). To determine the tension of the 2-0 tape, the total depth of the anchor insertion and the distance between anchors were calculated. The tension was adjusted, so that the 2-0 tape did not infiltrate the cartilage surface, which would cause cartilage damage during anchor fixation. After fixation, the specimen was mounted on a material-testing machine (Instron 4465; Instron Corp., Canton, MA) and was rigidly fastened in place using a specially designed clamp. The load cell size of material testing machine was $5 \mathrm{kN}$. A metal screw of $3.5 \mathrm{~mm}$ in diameter was inserted into the osteochondral fragment and then advanced perpendicular to the osteotomy plane, so that it could be clamped during tensile load testing, with the tensile load applied perpendicular to the osteotomy plane. Moreover, to reduce the osteochondral fragment anatomically, the subchondral bone of the osteochondral fragment was excavated, so that the screw head was completely buried in the subchondral bone (Figs 1 and 3).

The macroscopic appearance of the osteochondral fragment after testing and mode of failure were examined. Tensile loads at displacements of 1 and 2 $\mathrm{mm}$ and ultimate failure load were measured at a crosshead speed of $100 \mathrm{~mm} / \mathrm{min}$. A total of 6 biomechanical tests were performed in each group.

\section{Statistical Analysis}

The tensile strength and ultimate failure load of the three groups are shown as mean and standard deviations. Variable normality was determined using the 


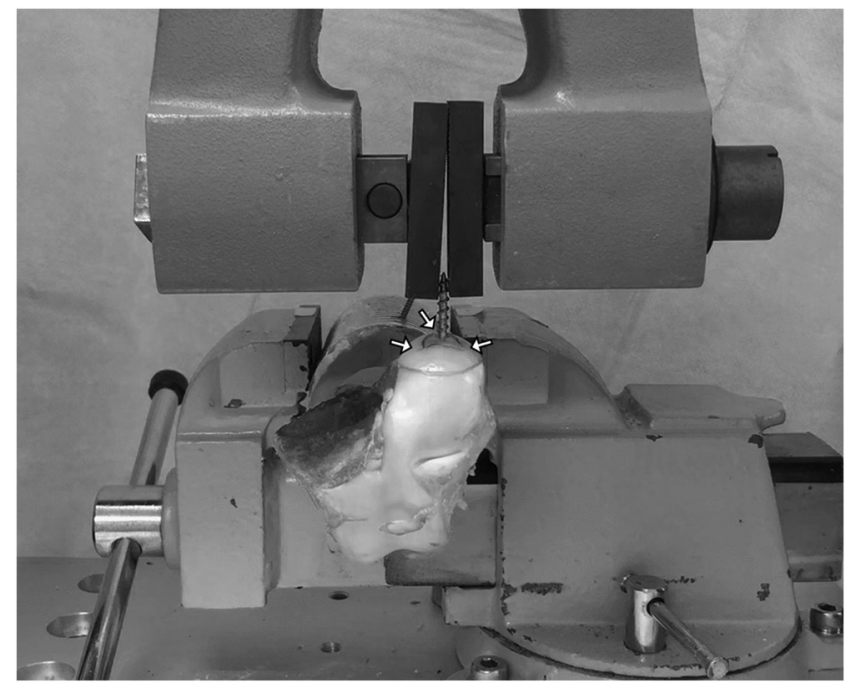

Fig 3. Setting of materials in the testing machine. A metal screw was inserted into the osteochondral fragment and clamped during tensile load testing. The tensile load is applied perpendicular to the osteotomy plane. The location of fixation device is indicated by arrows. This figure shows the testing in suture anchor group.

Shapiro-Wilk test. Biomechanical data were compared between groups using a one-way analysis of variance, with Tukey's honestly significant difference test. Results showed that the standard deviation of the overall ultimate failure load was $75.4 \mathrm{~N}$. When six specimens were used in each group, a post hoc power analysis revealed that type I error and its effect size were .854 and 1.065 , respectively $(P=.05)$. All statistical analyses were performed using SPSS version 27.0 (IBM Corp., Armonk, NY), and a $P$ value $<.05$ was considered statistically significant.

\section{Results}

There was no macroscopic osteochondral fragment fracture, articular cartilage crack, or clamped metal screw loosening after testing in all groups. The pull-out of the osteochondral fragment from the femoral condyle was observed in all specimens without breaking the osteochondral plug, bioabsorbable pin, suture anchor, and 2-0 tape.

The tensile load at a displacement of $1 \mathrm{~mm}$ was 30.8 $\pm 18.9 \mathrm{~N}$ in the osteochondral plug group, $49.8 \pm 28.4$ $\mathrm{N}$ in the PLLA group, and $55.3 \pm 20.2 \mathrm{~N}$ in the suture anchor group, indicating that there were no significant differences between groups $(P=.240)$ (Table 1). The tensile load at a displacement of $2 \mathrm{~mm}$ was significantly higher in the suture anchor group than in the osteochondral plug $(P=.002)$ and bioabsorbable pin $(P=.017)$ groups $(37.0 \pm 26.0 \mathrm{~N}$ in the osteochondral plug group, $52.8 \pm 24.1 \mathrm{~N}$ in the bioabsorbable pin group, and $100.4 \pm 21.5 \mathrm{~N}$ in the suture anchor group) (Table 1). Moreover, the ultimate failure load was higher in the suture anchor group than in the osteochondral plug and bioabsorbable pin groups $(P<.001$, both) $(40.8 \pm 25.8 \mathrm{~N}$ in the osteochondral plug group, $59.9 \pm 29.1 \mathrm{~N}$ in the bioabsorbable pin group, and $188.4 \pm 41.7 \mathrm{~N}$ in the suture anchor group) (Table 1).

\section{Discussion}

Single-pull destructive testing of a fixed articular osteochondral fragment with the force perpendicular to the articular surface, demonstrated no statistical difference in the tensile load to achieve $1-\mathrm{mm}$ displacement, but the load to achieve $2 \mathrm{~mm}$ displacement was significantly greater for the three-suture anchor-interlocking 2-0 tape constructs $(100.4 \pm 21.5 \mathrm{~N})$ than the dual 6-mm osteochondral plug fixation $(37.0 \pm 26.0 \mathrm{~N}$, $P=.002)$ and the four $1.5-\mathrm{mm}$ bioabsorbable pin fixation constructs $(52.8 \pm 24.1 \mathrm{~N}, P=.017)$. Additionally, the three suture anchor-interlocking 2-0 tape construct's mean single-pull failure load $(188.4 \pm 41.7 \mathrm{~N})$ was greater $(P<.001)$ than that of both the dual $6-\mathrm{mm}$ osteochondral plug fixation $(40.8 \pm 25.8 \mathrm{~N})$ and the four $1.5-\mathrm{mm}$ bioabsorbable pin fixation constructs $(59.9 \pm 29.1 \mathrm{~N})$.

Successful osteochondral fragment fixation outcomes mainly depend on the restoration of articular surface congruity and biological fixation. Initial fixation strength is an important factor of successful outcomes; however, it is affected by the properties or thickness of the osteochondral fragment.

Berlet et al. ${ }^{1}$ demonstrated an in situ fixation of an osteochondral fragment with an autogenous cylindrical osteochondral plug. Osteochondral plug fixation allowed an immediate and reliable whole tissue transfer, including the transfer of the viable hyaline cartilage, intact tidemark, subchondral bone, and bone. Miura et al. ${ }^{2}$ reported the clinical outcomes of 12 osteochondral fragment fixations for knee OCD using osteochondral plug technique. The interface between the osteochondral fragment and subchondral bone disappeared on magnetic resonance imaging in all patients at 3 months postoperatively. Yoshizumi et al. ${ }^{11}$ also reported a good postoperative course, demonstrating that union of the osteochondral plug and the entire OCD lesion was confirmed within 6 months postoperatively using the osteochondral plug technique. Although this procedure has the advantage of biological healing, donor site morbidity is possible.

Regarding bioabsorbable internal fixation for unstable knee OCD, Chun et al. ${ }^{12}$ reported favorable outcomes after bioabsorbable screw fixation in adolescents without complications due to fixation failure. In previous reports, ${ }^{13,14}$ although the number of patients was relatively small and there were some differences in the union rate depending on the age of patients, good 
Table 1. The Results of the Tensile Load at a Displacement of 1 and $2 \mathrm{~mm}$ and Ultimate Failure Load

\begin{tabular}{|c|c|c|c|c|c|c|}
\hline & \multicolumn{2}{|c|}{ Osteochondral Plug Group } & \multicolumn{2}{|c|}{ Bioabsorbable Pin Group } & \multicolumn{2}{|c|}{ Suture Anchor Group } \\
\hline & Mean \pm SD (range) & $95 \% \mathrm{CI}$ & Mean \pm SD (range) & $95 \%$ CI & Mean \pm SD (range) & $95 \% \mathrm{CI}$ \\
\hline $\begin{array}{l}\text { Tensile load at a } \\
\text { displacement } \\
\text { of } 1 \mathrm{~mm}(\mathrm{~N})\end{array}$ & $30.8 \pm 18.9(6.8-63.8)$ & $9.0-52.6$ & $49.8 \pm 28.4(7.0-93.9)$ & $17.1-82.4$ & $55.3 \pm 20.2(23.8-75.7)$ & $32.0-78.6$ \\
\hline $\begin{array}{l}\text { Tensile load at a } \\
\text { displacement } \\
\text { of } 2 \mathrm{~mm}(\mathrm{~N})\end{array}$ & $37.0 \pm 26.0(12.0-82.4)$ & $7.0-67.0$ & $52.8 \pm 24.1(13.0-87.3)$ & $25.0-80.5$ & $100.4 \pm 21.5(72.4-134.7)^{* \dagger}$ & 75.7-125.1 \\
\hline
\end{tabular}

clinical results have been reported. In contrast, low rates of clinical healing and high complication rates after bioabsorbable fixation among skeletally mature patients were suggested. ${ }^{15}$ Nguyen et al. ${ }^{16}$ conducted a magnetic resonance imaging study to investigate the knee joint of children following osteochondral fragment fixation using bioabsorbable nails. They revealed that nail breakage was observed in 26 of 58 patients $(45 \%)$, with a median time of 6.5 months between operation and the first study, in which a broken nail was observed. Among patients with nail breakage, those who were diagnosed with a new meniscal tear, all broken nails were situated at the site of the torn meniscus. In addition, nail breakage was observed in $57 \%$ of cases of patients diagnosed with a new cartilage injury. They concluded that the high prevalence of nail breakage was independent of skeletal maturity and the total number of nails used. Although this procedure does not induce donor site morbidity, there is a risk of insufficient fixation strength and secondary injury to the intra-articular structure due to implant breakage or backout.

Recently, some surgical osteochondral fragment fixation techniques using suture anchors were proposed. ${ }^{8-10}$ Ishibashi et al. ${ }^{9}$ demonstrated the suture anchor fixation technique, which is used in this experiment. They suggested that this technique could fix and press down over a wide area in a triangular structure without suture tying. The results of this biomechanical study supported their new technique as the ultimate failure load was significantly higher in the suture anchor group than in the other two techniques.

In this study, there was no significant difference between groups in terms of tensile strength at a displacement of $1 \mathrm{~mm}$; however, the suture anchor group demonstrated a significantly higher strength at a displacement of $2 \mathrm{~mm}$. This may be due to the suture anchor structure. Because this suture anchor has a separate eyelet and anchor, slight differences in anchor insertion depth may affect tensile strength. This suggests that it is important to insert the anchor at a depth at which it makes full contact with the eyelet; however, if the anchor is inserted deeper than required, the suture will be overtensioned, raising a risk of osteochondral fragment surface damage. Therefore, it is essential to drill and insert anchors to an appropriate depth clinically.

\section{Limitations}

There are several limitations to this study. First, this was a time 0 study that did not consider cyclic loading. Osteochondral plug fixation has advantages when considering the effects of biological unions. Therefore, there may be patients in whom osteochondral plug fixation is indicated, depending on the condition of the osteochondral fragment and the remaining subchondral bone. The second limitation of this study was that the tensile strength when using other fixation devices or other types of fixation has not been examined. In addition, only perpendicular force was applied to the osteochondral fragment; therefore, the sharing force generated in vivo was not reproduced. Since failure mechanism in clinical situation is considered to be caused by sharing force to the osteochondral fragment, it is unclear whether the fixation strength in this result of this study is directly linked to clinical outcome. The third limitation was the experiment environment, including a room temperature or not performing the tensile test in an aqueous environment.

\section{Conclusions}

Single-pull destructive testing of a fixed articular osteochondral fragment with the force perpendicular to the articular surface, demonstrated no statistical difference in the tensile load to achieve $1-\mathrm{mm}$ displacement, but the load to achieve 2 -mm displacement was significantly greater for the three suture anchorinterlocking 2-0 tape constructs than the dual 6-mm osteochondral plug fixation and the four $1.5-\mathrm{mm}$ bioabsorbable pin fixation constructs. Additionally, the three suture anchor-interlocking 2-0 tape construct's mean single-pull failure load was greater than other two fixation procedures. 


\section{References}

1. Berlet GC, Mascia A, Miniaci A. Treatment of unstable osteochondritis dissecans lesions of the knee using autogenous osteochondral grafts (mosaicplasty). Arthroscopy 1999;15:312-316.

2. Miura K, Ishibashi Y, Tsuda E, Sato H, Toh S. Results of arthroscopic fixation of osteochondritis dissecans lesion of the knee with cylindrical autogenous osteochondral plugs. Am J Sports Med 2007;35:216-222.

3. Adachi N, Motoyama M, Deie M, Ishikawa M, Arihiro K, Ochi M. Histological evaluation of internally-fixed osteochondral lesions of the knee. J Bone Joint Surg Br 2009;91: 823-829.

4. Adachi N, Deie M, Nakamae A, Okuhara A, Kamei G, Ochi M. Functional and radiographic outcomes of unstable juvenile osteochondritis dissecans of the knee treated with lesion fixation using bioabsorbable pins. J Pediatr Orthop 2015;35:82-88.

5. Kocher MS, Czarnecki JJ, Andersen JS, Micheli LJ. Internal fixation of juvenile osteochondritis dissecans lesions of the knee. Am J Sports Med 2007;35:712-718.

6. Matsusue Y, Nakamura T, Suzuki S, Iwasaki R. Biodegradable pin fixation of osteochondral fragments of the knee. Clin Orthop Relat Res 1996;322:166-173.

7. Yonetani Y, Matsuo T, Nakamura N, et al. Fixation of detached osteochondritis dissecans lesions with bioabsorbable pins: clinical and histologic evaluation. Arthroscopy 2010;26:782-789.

8. Chernchujit B, Artha A. Osteochondritis dissecans of the knee: Arthroscopic suture anchor fixation. Arthrosc Tech 2020;9:e1203-e1209.
9. Ishibashi Y, Kimura Y, Sasaki S, Sasaki E, Takahashi A. Internal fixation of osteochondritis dissecans using PushLock suture anchors. Arthrosc Tech 2021;10: e705-e709.

10. Vogel LA, Fitzsimmons KP, Lee Pace J. Osteochondral fracture fixation with fragment preserving suture technique. Arthrosc Tech 2020;9:e761-e767.

11. Yoshizumi Y, Sugita T, Kawamata T, Ohnuma M, Maeda S. Cylindrical osteochondral graft for osteochondritis dissecans of the knee: A report of three cases. Am J Sports Med 2002;30:441-445.

12. Chun KC, Kim KM, Jeong KJ, Lee YC, Kim JW, Chun CH. Arthroscopic bioabsorbable screw fixation of unstable osteochondritis dissecans in adolescents: Clinical results, magnetic resonance imaging, and secondlook arthroscopic findings. Clin Orthop Surg 2016;8: 57-64.

13. Din R, Annear P, Scaddan J. Internal fixation of undisplaced lesions of osteochondritis dissecans in the knee. J Bone Joint Surg Br 2006;88:900-904.

14. Dines JS, Fealy S, Potter HG, Warren RF. Outcomes of osteochondral lesions of the knee repaired with a bioabsorbable device. Arthroscopy 2008;24:62-88.

15. Millington KL, Shah JP, Dahm DL, Levy BA, Stuart MJ. Bioabsorbable fixation of unstable osteochondritis dissecans lesions. Am J Sports Med 2010;38: 2065-2070.

16. Nguyen JC, Green DW, Lin BF, Endo Y. Magnetic resonance evaluation of the pediatric knee after arthroscopic fixation of osteochondral lesions with biodegradable nails. Skeletal Radiol 2020;49:65-73. 